Nachruf

Z. Epileptol. 2020 · 33:177-178

https://doi.org/10.1007/s10309-020-00314-y

Online publiziert: 10. März 2020

(c) Springer Medizin Verlag $\mathrm{GmbH}$, ein Teil von Springer Nature 2020

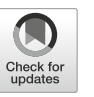

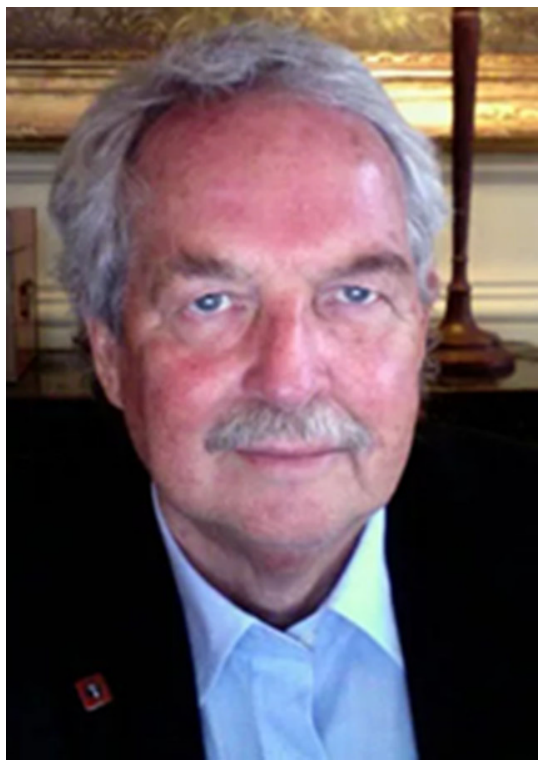

Abb. $1 \Delta$ Dieter Schmidt. (Abbildung aus [1] mit freundl. Genehmigung)

Am 08.09.2019 ist Dieter Schmidt (• Abb. 1) nach langer Zeit geduldig, am Ende schließlich schwer zu ertragender Krankheit im Alter von 72 Jahren in aller Stille in Berlin zu Hause gestorben. Mit ihm hat die deutschsprachige klinische Epileptologie einen ihrer - v. a. international - renommiertesten Vertreter der letzten Jahrzehnte verloren.

Dieter Schmidt wurde am 28.04.1947 in Bad Schwalbach im Taunus geboren und konnte bei entsprechenden Gelegenheiten durchaus auch auf „Hessisch“

Unter Verwendung eines Textes von Wolfgang Löscher für die ILAE-Website, Epilepsia Open und Epilepsy \& Behavior [1]

\author{
Günter Krämer ${ }^{1} \cdot$ Wolfgang Löscher ${ }^{2} \cdot$ Christian Erich Elger $^{3} \cdot$ Matti Sillanpää $^{4}$ \\ ${ }^{1}$ Neurozentrum Bellevue, Zürich, Schweiz \\ ${ }^{2}$ Tierärztliche Hochschule Hannover, Hannover, Deutschland \\ ${ }^{3}$ Beta Klinik Bonn, Bonn, Deutschland \\ ${ }^{4}$ Universität von Turku, Turku, Finnland
}

\title{
Nachruf auf Professor Dr. med. Dieter Schmidt (28.04.1947-08.09.2019)
}

kommunizieren. Sein Studium absolvierte er in Heidelberg, anschließend dort auch die neurologische Facharztweiterbildung bei Dieter Janz (damals Oberarzt; 1920-2016) an der von Heinz Gänshirt (1919-1991) geleiteten Neurologischen Universitätsklinik. Im Jahr 1973 wechselte er zusammen mit Janz, der einen Ruf auf das Ordinariat für Neurologie am Klinikum Charlottenburg der dortigen Freien Universität erhalten hatte, nach West-Berlin. Dort habilitierte er sich 1982 und wurde zum Privatdozenten ernannt. Aus dieser Zeit stammen u. a. auch Arbeiten zusammen mit Janz zum Versagen hormoneller Kontrazeptiva unter Einnahme enzyminduzierender Antiepileptika (z. B. [2]).

Im Jahr 1985 wechselte Dieter Schmidt als Oberarzt und Leiter des Epilepsiebereichs an die Neurologische Klinik der Ludwig-Maximilians-Universität in München zu Thomas Brandt, wo er auch zum außerplanmäßigen Professor ernannt wurde. $\mathrm{Zu}$ seinen dortigen Mitarbeitern zählten u. a. Bernhard Steinhoff (jetzt Ärztlicher Direktor des EpilepsieZentrums Kork) und Stefan Stodieck (jetzt Chefarzt der Epileptologie am Evangelischen Krankenhaus Alsterdorf und Ärztlicher Leiter des Norddeutschen Epilepsiezentrums Hamburg).

Im Jahr 1989 wurde Dieter Schmidt nach der Emeritierung von Dieter Janz im Vorjahr als ordentlicher Professor und Ordinarius für Neurologie nach Berlin zurückberufen. Eine Mitarbeiterin dort war u.a. die leider sehr früh verstorbene Sibylle Ried (1956-2000), die 1995 an das Schweizerische Epilepsie-Zentrum in Zürich wechselte. Bereits nach 3 Jahren, 1992, erkrankte er kritisch. Die Natur der Erkrankung war mit der anspruchsvollen Tätigkeit als Direktor einer Neurologischen Universitätsklinik nicht vereinbar, weshalb er vorzeitig den Ruhestand antrat.

Wer Dieter Schmidt kannte, weiß, dass er trotz der gesundheitlichen Belastung nicht untätig bleiben wollte und konnte. Er gründete eine „Forschungsgruppe Epilepsie" und war sowohl national als auch international sehr aktiv. Sein analytisches Talent ermöglichte es ihm, Daten zielorientiert aufzuarbeiten und in klare Konzepte zu strukturieren. Innerhalb der Deutschen Gesellschaft für Epileptologie war er Mitglied verschiedener Kommissionen, u. a. zu Generika [3], oder federführender Autor von Expertentreffen zu neuen Antiepileptika [4]. Er war ein oft eingeladener Redner auf Kongressen, Symposien und Workshops. Unter anderem begründete Dieter Schmidt zusammen mit Wolfgang Löscher die erstmals 2002 in Philadelphia abgehaltene Workshopreihe "New Horizons in the Development of Antiepileptic Drugs".

Dieter Schmidt hat mit jedem von uns gemeinsam mehrere Artikel oder Bücher publiziert, war (zusammen mit Wolfgang Löscher) Gründungsherausgeber der Zeitschrift Epilepsy Research und später (zusammen mit Aristea Galanopoulou) des Journals Epilepsia open der Internationalen Liga gegen Epilepsie (ILAE). Er hat, zuletzt zusammen mit Christian Elger, mehrere Lehrbücher 
der Erwachsenen-Epileptologie verfasst. Seine letzten wissenschaftlichen Aktivitäten bestanden in einer Serie zusammen mit Matti Sillanpää verfassten Artikeln u. a. in Brain [5-8], Nature Reviews Neurology und Epilepsia mit der Analyse populationsbasierter Studien zum Langzeitverlauf kindlicher Epilepsiesyndrome. Sein letztes, gemeinsam mit Simon Shorvon publiziertes Buch hatte den bewusst provokanten Titel „The End of Epilepsy?" Insgesamt war er Koautor oder Mitherausgeber von 21 Büchern [9-29]!

Ein wesentliches Anliegen war ihm, seine Kolleginnen und Kollegen in der Epileptologie sinnvoll weiterzubilden. Zahlreiche Fortbildungsveranstaltungen tragen seine Handschrift. Beim europäischen Epilepsiekongress gründete er einen Kurs, der rasch zur Tradition wurde. Insbesondere legendär waren seine meist sehr interaktiven Kurse. Mit spritzigem, geistreichem Humor konnte er die Teilnehmer fesseln, sodass Person und Inhalt unvergesslich blieben.

Persönlich war Dieter Schmidt wie jeder von uns ein Mensch mit Stärken und Schwächen. Er war ein Freund der direkten und klaren Ansprache, gleichwohl verbarg sich hinter der manchmal etwas rauen Schale ein oft sensibler Kern. Wer ihn persönlich gut kannte, sah, dass viele der oft unangemessenen Kränkungen deutliche Spuren hinterließen. Er hatte die seltene Eigenschaft, „out of the box“ denken zu können, was viele seiner innovativen und provokanten Ideen erklärt. Mit manchen seiner Publikationen hat er sich auch nicht unbedingt allseits beliebt gemacht, so z. B. mit seiner trotz vieler anfänglicher Widerstände später im Prinzip bestätigten Metaanalyse der Chance einer dauerhaften Anfallsfreiheit ohne Einnahme von Antiepileptika nach epilepsiechirurgischen Eingriffen, die mit $30 \%$ deutlich niedriger liegt als häufig propagiert wird [30]. Wer damit umgehen konnte, hatte in ihm einen äußerst kompetenten und zuverlässigen Ansprechpartner und Kollegen. Anfragen beantwortete er prompt und zuverlässig, wo auch immer er gerade auf der Welt unterwegs war. Er war ein kreativer Mensch, der immer noch neue Standpunkte und Aspekte entdeckte, wenn in einem Untersuchungsprojekt alles analysiert zu sein schien, und war klar und konstruktiv in seinen Vorschlägen.

Dieter Schmidt hat die internationale klinische Epileptologie nachhaltig mitgeprägt. Von den zahlreichen Auszeichnungen, die er erhielt, seien hier der "Ambassador for Epilepsy“ durch die ILAE und das IBE 1983 sowie der Alfred-Hauptmann-Preis des Epilepsiekuratoriums 1984 hervorgehoben. Wir vermissen ihn, und unsere Gedanken sind bei seiner Frau.

\section{Korrespondenzadresse}

\section{Dr. med. Günter Krämer}

Neurozentrum Bellevue

Theaterstr. 8, 8001 Zürich, Schweiz

g.kraemer@epilepsie-med.de

\section{Literatur}

1. Löscher W (2020) In memoriam Dieter Schmidt. Epilepsy Behav 103:106583

2. Janz D, Schmidt D (1974) Anti-epileptic drugs and failure of oral contraceptives (letter). Lancet 1(7866):1113

3. Krämer G, Dennig D, Schmidt D et al (2006) Generika in der Epilepsietherapie Was ist zu beachten? ZEpileptol 19:1-5

4. Schmidt D, Stöhr T, Uebachs M et al (2008) Lacosamid (Vimpat ${ }^{\circledR}$ ): Bericht eines Expertentreffens zu einem neuen Medikament zur Zusatzbehandlung fokaler Anfälle.ZEpileptol 21:180-189

5. Sillanpää M, Schmidt D (2006) Natural history of treated childhood-onset epilepsy: prospective, long-term population-based study. Brain 129:617-624

6. Sillanpää M, Schmidt D (2008) Seizure clustering during drug treatment affects seizure outcome and mortality of childhood-onset epilepsy. Brain 131:938-944

7. Sillanpää M, Schmidt D (2009) Early seizure frequency and aetiology predict long-term medical outcome in childhood-onset epilepsy. Brain 132:989-998

8. Sillanpää M, Schmidt D (2012) Is incident drugresistance of childhood-onset epilepsy reversible? A long-term follow-up study. Brain 135:2256-2262

9. Johannessen $\mathrm{SI}$, Morselli PL, Pippenger $\mathrm{CE}, \mathrm{Ri}-$ chens A, Schmidt D, Meinardi H (Hrsg) (1980) Antiepileptic therapy. Advances in drug monitoring. Workshop on the determination of antiepileptic drugs in body fluids, Oslo, June 1979. Raven Press, New York

10. Schmidt D (1984) Behandlung der Epilepsien. Medikamentös - Psychosozial - Operativ. Thieme, Stuttgart-New York

11. Janz D, Dam M, Richens A, Bossi L, Helge $H$, Schmidt D (Hrsg) (1982) Epilepsy, pregnancy, and the child. Raven Press, New York

12. Schmidt D, Seldon L (1982) Adverse effects of antiepileptic drugs. Raven Press, New York

13. Schmidt D, Morselli PL (Hrsg) (1986) Intractable epilepsy: experimental and clinical aspects. L.E.R.S. Monograph series, Bd. 5. Raven Press, New York
14. Schmidt D, Wauquier $A$, Meinardi $H$, van den Bussche G (Hrsg) (1986) International workshop on flunarizine in epilepsy. Beerse, May 24, 1985. KPR, Brussels

15. Schmidt D (2012) Epilepsien. Fragen und Antworten, 8. Aufl. W. Zuckschwerdt, München - Bern Wien - San Francisco

16. Schmidt D, Leppik I (Hrsg) (1988) Compliance in epilepsy. Epilepsy research, Bd. 1. Elsevier, Amsterdam-New York-Oxford

17. Schmidt D (1992) Taschenatlas Epilepsien. W. Zuckschwerdt, München - Bern - Wien - New York

18. Schmidt D (1996) Pharmakotherapie der Epilepsien. Behandlungsstrategien und Klinische Pharmakologie, 3. Aufl. W. Zuckschwerdt, München-Bern-Wien-New York

19. Schmidt D (1993) Epilepsie und epileptische Anfälle. Thieme, Stuttgart-New York

20. Krämer G, Schmidt D (Hrsg) (1994) Vigabatrin. Pharmakologie - Wirksamkeit - Verträglichkeit. Springer, Berlin-Heidelberg-New York

21. Epilepsie SD (1997) Diagnostik und Therapie für Klinikund Praxis. Schattauer, Stuttgart-New York

22. Schmidt D (1998) Drug trials in epilepsy. A physician's guide. M. Dunitz, London

23. Schmidt D, Tatum W, Schachter S (2018) Common pitfalls in epilepsy. Case-based learning. Cambridge University Press, Cambridge - New York Port Melbourne, et al

24. Schmidt D, Elger CE (2005) Praktische Epilepsiebehandlung. Praxisorientierte Diagnose und Differentialdiagnose, rationale Therapiestrategien und handlungsorientierte Leitlinien, 3. Aufl. Thieme, Stuttgart-New York

25. Schmidt D, Schachter SC (Hrsg) (2000) Epilepsy. Problem solving in clinical practice. M. Dunitz, London

26. Schachter CS, Schmidt D (Hrsg) (2003) Vagus nerve stimulation, 2. Aufl. M. Dunitz, London-New York

27. Schmidt D, Schachter S (Hrsg) (2019) One hundred case studies in epilepsy. CRC Press, Boca Raton London-New York

28. Elger CE, Schmidt D (2007) Aktuelle Epilepsietherapie: kurzgefasst, 4. Aufl. W. Zuckschwerdt, München

29. Schmidt D, Shorvon S (2016) The end of epilepsy? A history of the modern era of epilepsy research 1860-2010. Oxford University Press, Oxford - New York

30. Schmidt D, Baumgartner C, Löscher W (2004) The chance of cure following surgery for drug-resistant temporal lobe epilepsy. What do we know and do we need to revise our expectations? Epilepsy Res 60:187-201 